\title{
Presentation of a novel real-time production supply concept with cyber-physical systems and efficiency validation by process status indicators
}

\author{
Haci Bayhan ${ }^{1} \cdot$ Matthias Meißner $^{2} \cdot$ Pascal Kaiser $^{1} \cdot$ Maria Meyer $^{1} \cdot$ Michael ten Hompel $^{3}$
}

Received: 29 January 2020 / Accepted: 28 April 2020 / Published online: 16 May 2020

(C) The Author(s) 2020

\begin{abstract}
Industry 4.0, digitization, and Internet of Things enable companies to react quickly and flexibly to market changes. The objective is the production of customized products at the cost of a mass-produced product. The widespread entry of cyberphysical systems enables a networking of all entities. The introduction of cyber-physical systems in production and logistic systems dissolves existing rigid structures and as a result versatile cyber-physical production systems occur. To support the flexibility of the cyber-physical production systems, the production supply needs to be adapted. For this reason, this paper proposes a novel universal production supply concept. This concept introduces a decentralized controlled supply. It is executed by several cyber-physical system entities, which are represented by software agents. These agents negotiate autonomously with each other. The novel concept was implemented in a research lab and evaluated quantitatively and qualitatively. For the quantitative evaluation, the efficiency of the novel concept is evaluated and compared with a kanban supply. An innovative key performance indicator system called process status indicators evaluates the efficiency. The result of this indicator system states that the novel concept is more efficient than the kanban supply.
\end{abstract}

Keywords Cyber-physical systems · Cyber-physical production systems $\cdot$ Real-time production supply concept · Decentralized production supply

\section{Introduction}

Manufacturing companies have to handle the challenge to adapt to market dynamics and technological trends on time. Customized products and shorter lead times increase significantly the complexity of processes especially in the

Matthias Meißner

matthias.meissner@tu-dortmund.de

Haci Bayhan

haci.bayhan@tu-dortmund.de

1 Chair of Material Handling and Warehousing, TU Dortmund University, Joseph-von-Fraunhofer Straße 2-4, 44227 Dortmund, Germany

2 Institute of Energy Systems, Energy Efficiency and Energy Economics, TU Dortmund University, Emil-Figge-Straße 70, 44227 Dortmund, Germany

3 Fraunhofer Institute for Material Flow and Logistics IML, Joseph-von-Fraunhofer Straße 2-4, 44227 Dortmund, Germany supply chain [1]. Additionally, shorter response and order lead time confront complex supply networks. This affects the failure sensitivity of the system. The results are forecasting inaccuracies and planning uncertainties. Therefore, the industrial internet consortium and industry 4.0 discuss robust systems and processes to make the complexity manageable. Key elements are cyber-physical systems (CPS) acting autonomously. The entities use the Internet of Things to connect and collaborate with each other [2]. Intelligent software enables the autonomy in CPS and in this concept it is supported by a decentralized decision-making. The use of CPS dissolves rigid structures within production and logistic systems. As a result, versatile cyber-physical production systems (CPPS) arise. To support the flexibility of CPPS, an adjustment of the production supply to a realtime production supply is necessary. The basic idea is that software agents perform autonomously the decision and routing of logistic objects in a dynamic environment. The objective is an efficient, self-controlling, and need-based production supply. The main challenge of a real-time production supply in CPPS is that the production sequence and 
thus the actual part demand in location and time are initially unknown. Instead of a defined production and order sequence, agents control the decision which will be the next production step. The agent decides the order processing sequence ad hoc based on current system states (e.g., inventory availability) and occurring events (e.g., workstation failures). The gained flexibility can only generate a benefit, if the processing sequence of the object is coordinated and optimized. This effects especially the production supply of customized parts [3, 4]. The production supply must handle very short lead times. The available lead time of the workstation complies with the design of the production system and the relation between transport and process time. The workstation will experience part shortages, if the lead time is to short, because the parts will not arrive on time. This leads to an increased cycle time, and in critical cases, delivery dates cannot be met. For a decrease of the lead time, there is the possibility of storing the parts close to the workstation. Because the precise demand is not known, every part that might be required has to be in stock. Depending on the product, order quantity, and size of the production system, this results in high inventory, high warehousing costs, and high capital lockup, and therefore, it is not practical. A production supply concept does not exist that not only enables the flexibility in CPPS but also makes the CPPS efficient in terms of low inventories and high utilization. For this reason, the authors developed a concept for a decentralized controlled real-time production supply in CPPS at the Chair of Material Handling and Warehousing at the TU Dortmund.

The main objective of this paper is to propose a novel production supply concept for cyber-physical production systems. This concept will be implemented in the research lab, and to evaluate the efficiency of the novel concept, a key performance indicator system compares it with a production supply with a classical concept. The key performance indicator system that is used is called "process status indicators" and is able to describe the efficiency of processes or process chains in detail. The efficiency is represented as a function of an arbitrary number of input and output variables. Furthermore, two different production systems that execute the same process can be directly set in relation to each other. In this way, the efficiency of both systems can be directly compared.

The remainder of the paper is organized as follows. The second section describes the state of the art. In the third section, the novel real-time production supply concept (realProS) is introduced with all its aspects. The fourth section presents the validation of the novel concept, a classical production supply concept, and an energy efficiency comparison between these concepts. The last section summarizes the results of the comparison, draws a conclusion, and shows future research work.

\section{State of the art}

New research in the field of production supply is limited. Therefore, this section shows the concepts that were developed without CPS, in the remainder of the paper called classical concepts as well as current research, which focuses on new technology.

On the one hand, there are the classical concepts such as kanban or milk run. In kanban, the production supply is organized based on the pull-principle. The resupply is consumption-controlled; this means that the concept achieves a high degree of synchronization between the demand and the production $[5,6]$. A milk run system is used for the material transport. The milk run supplies different staging areas with different materials on one trip [7]. Generally, it is evident that consumption-controlled material-supply strategies (e.g., in kanban), high inconsistency of the required quantity at each single production module, dynamic cycle times, and flexible transportation routes lead to this strategy being only applicable with uneconomic inventories in classic material-supply strategies.

Diversely, material supply in demand-controlled material-supply strategies works after the pull-principle. The central control unit is responsible for the trigger of the material supply. Based on the production program, it is exactly and deterministically determined which material is to be provided where at any point in time, which is not suitable for CPPS [5]. On the other hand, there are some researches regarding the development of a decentralized production supply in a production system. A brief overview of some of the researches is given in the following.

Bortolini et al. [8] propose a general framework for the assembly system design in the Industry 4.0 era. This research focuses on enabling a real-time communication by equipping all entities with sensors. Instead, the primary focus of realProS is on the supply of production parts in consideration of the 6R: delivery of the right product at the right time, place, amount, quality, and costdespite the increasing number of customized parts, wide variety, minimum inventory, limited space, etc. realProS is a generally valid concept which is explicitly adapted for production supply. Thus, the general framework does not focus on the production supply. It proposes to optimize the stock and automatically create refill orders through an intelligent storage management.

Wang et al. [9] propose a concept consisting of three modules. The first module is the physical shop floor. This module is responsible to create an intelligent shop floor with CPS technologies. The second module is the shop floor digital twin to model the physical shop floor and the third module is a proactive material handling based on prediction of future status of the system. This concept 
enables an optimized decision-making through CPS but relies on known logistic concepts. realPros also utilize the algorithm for ideal decision-making with regard to choosing the entities for pending tasks (optimal robot, material, workstation, employee, etc.). A difference is that these decisions are made just in time (i.e., not in form of a "digital twin" - thus reducing the costs). The second difference is that realPros solely deals with production supply and not with intelligent shop floor.

The research project SMART FACE [10] focuses on a decentralized control of flexible assembly stations. Automated guided vehicles (AGVs) are used for the transportation between the stations. The research prioritizes the production planning and does not focus on the production supply tasks. The analysis of current literature and personal exchange among colleagues, who were active in SMART FACE, made clear that production supply was only rudimentarily considered with respect to the completeness of the process.

The KARIS PRO [11] project developed an autonomous intralogistics system in the production environment. KARIS PRO is an AGV that works without any additional hardware such as a warehouse management system. Because of the complete decentralization, the AGV has to create transport orders itself. Therefore, the focus of this research is on the method of creating these transport orders. However, the common mistake spotted in existing research projects is that many projects which deal with Industry 4.0 respectively CPPS (developing hardware) are unconcerned about a suitable concept for the developed hardware. Thereby, the full potential of Industry 4.0 or CPS technologies cannot be fully tapped. Here again, a generally valid concept is lacking.

Vojdani and Knopp [12] developed an adaptive production supply concept on the basis of an agent-based transport management. The focus in this research is on solving shortterm planning problems such as transportation planning and the related production supply. To solve this problem, Vojdani and Knopp designed a concept that changes the production supply strategy based on the current situation without any changes to the infrastructure. Decentralized software agents realize this concept. These problems are ought to be countered with a situation-specific exchange of material-supply strategies, which do not require any infrastructure modification. The objective of this concept is to provide the selection of an ideal, situation-specific strategy. The basis for the selection is key indicators retrieved by monitoring the current material-supply strategy. This monitoring, including assessment and rescheduling, is to be implemented decentralized with software agents. The difference in this case is that already existing materialsupply strategies are made use of. The new concept develops an innovative approach for production supply with regard to CPPS. As mentioned above, classic production supply strategies are unsuitable for CPPS.

Summarized, all the researches mentioned above focus only on one aspect of the production supply. In [12], the focus is set mainly on adapting existing classical production supply concepts with software agents. Next in [11], the focus is solely on the hardware. The concepts of [8] and [9] deal mainly with the control of production while the production supply is hardly considered and [10] is specific for an adaptive automotive production in a decentralized production supply. None of the above brings all the different aspects together and creates one single concept, which is the objective of the concept presented in this paper.

\section{3 realProS: real-time production supply concept for cyber-physical production systems}

The real-time production supply concept for cyber-physical production systems realProS is a novel production supply concept. It was created to handle the previous described development in the manufacturing environment and to close the gap of the already existing research. This section presents the novel concept and performs a qualitative evaluation of the concept. This concept only considers the production supply and no other required resources for the production.

\subsection{Structure of the concept}

realProS consists of several CPPS entities (e.g., workstations and AGVs) that communicate with each other. The communication is decentralized structured, so every entity can make decisions. For this decision-making, all entities are represented by software agents. In general, these agents' use offers for all multi-criteria negotiations. The offers contain different criteria for the decision-making. Figure 1 shows the whole communication relationship between the CPPS entities. The following paragraphs describe this relationship and the subsections give a more detailed description of each task in the communication.

The communication begins when a workstation agent (WSAgent) receives a production order from the production planning. Then, the WS-Agents start negotiating to find the most suitable one for the production order. Therefore, every WS-Agent creates an offer. In order to do this, every WSAgent runs through the following process that is shown as an example for one WS-Agent in the following paragraphs. Section 3.1.1 describes the production order in more detail.

The first step after a WS-Agent receives a production order is to check if a picking order is necessary. When necessary, the WS-Agent creates a picking order and sends 


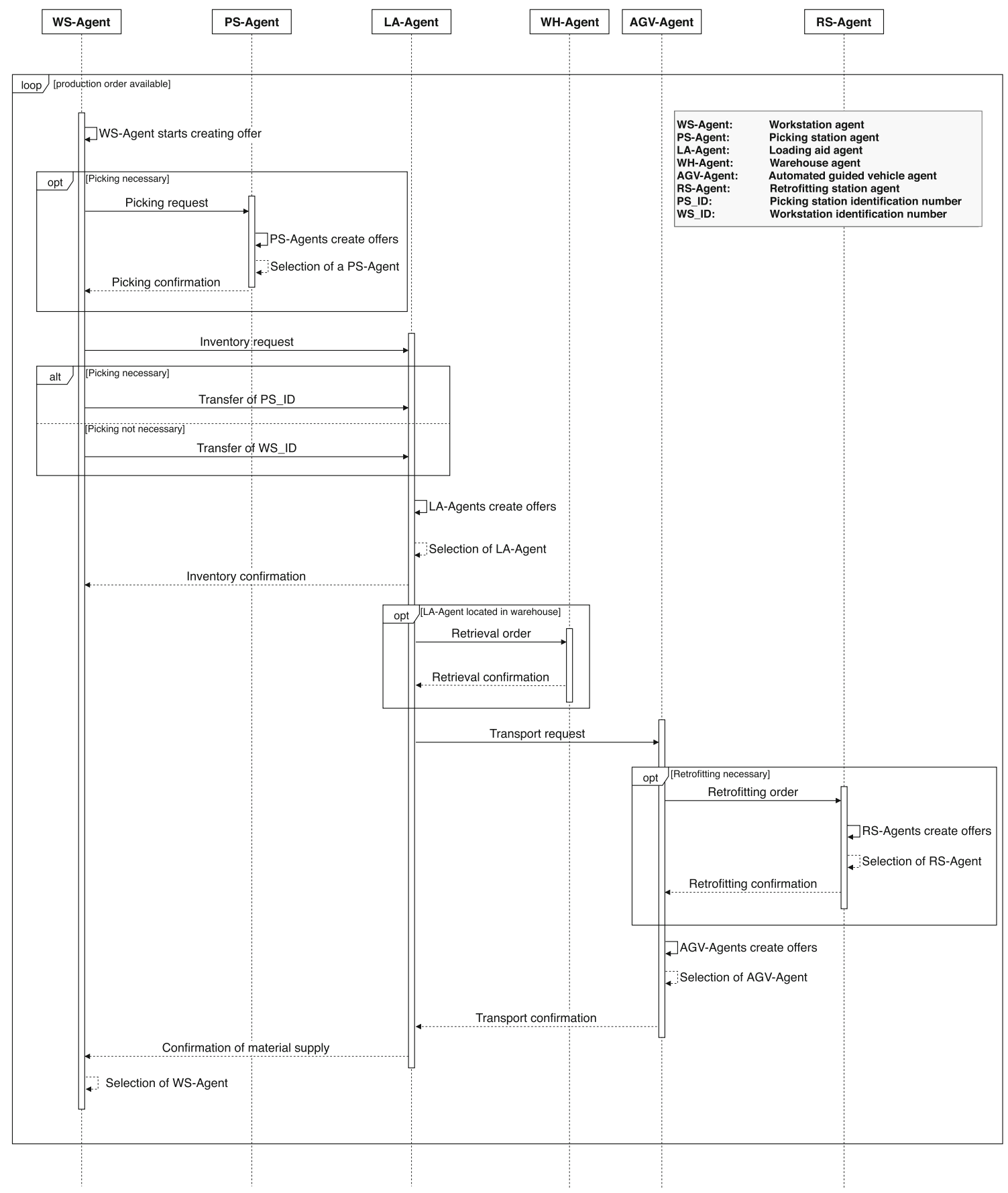

Fig. 1 Sequence diagram of realProS

it to the picking station agent (PS-Agent). Otherwise, the WS-Agent skips this step. PS-Agents that are able to fulfill the request create an offer and negotiate with each other to select the most suitable among them. The selected PS-Agent sends a confirmation back to the WS-Agent. The whole picking process is explained in Section 3.1.2 in more detail.

Afterwards or if a picking order is not necessary, the WS-Agent creates and sends an inventory request to the loading aid agent (LA-Agent). With the inventory request, the WS-Agent includes the destination for the transfer point.
This transfer point can be the workstation itself or the selected picking station. Every LA-Agent that meets the criteria creates an offer and the LA-Agents negotiate, which is the most suitable one. The selected LA-Agent sends a confirmation back to the WS-Agent. Section 3.1.3 states this process more precisely.

Then, the selected LA-Agent creates a warehouse order and sends it to the warehouse agent (WH-Agent) if necessary. This order is only created if the loading aid is located in a warehouse. The WH-Agent confirms the 
retrieval and sends back the confirmation together with the retrieval position. Section 3.1.4 specifies this process.

After this, the LA-Agent creates a transport order and sends it with its criteria to the automated guided vehicle agents (AGV-Agents). The AGV-Agents then check the criteria of the order. If a retrofitting is necessary to fulfill the criteria of the transport order, the AGV-Agent creates and sends a retrofitting order. In this case, the retrofitting station agents (RS-Agents) create offers and negotiate, which is the most suitable one. The selected RS-Agent sends a retrofitting confirmation back to the AGV-Agent. Section 3.1.5 describes this process in more detail.

Afterwards, all other AGV-Agents that are able to fulfill the order create an offer. Then, the AGV-Agents negotiate which is the most suitable one and the selected AGVAgent sends a transport confirmation to the LA-Agent. In Section 3.1.6, this process is elaborated in more detail. As soon as the LA-Agent receives the transport confirmation, it sends a production supply confirmation to the WS-Agent. This message contains the arrival time and the availability of the material.

To handle errors within the system every message contains a unique sequence number and every entity can create error messages. The section's sequence number and error message describe the interaction between the sequence number and an error message. This communication relationship is an iterative process. That means it repeats itself as long as there are production orders in the system.

\subsubsection{Production order}

The production planning sends a production order to all WS-Agents. This order includes which product in which quantity has to be produced. This message also includes the product identification number (ID), the latest date for completion, and the earliest start date of the product. The WS-Agents use the product ID to identify the required materials and their quantity. Additionally, production-specific information can be added, for example, the processing sequence. It is assumed that every production order can be processed, if it is communicated to the WS-Agent.

\subsubsection{Picking order}

A WS-Agent creates and sends the picking order. Receivers of this message are all PS-Agents present in the system. The message includes the picking request, the time slot, information about the material, the quantity, and the material ID. This material ID enables the picking stations to check if the material in the supplied loading aid is the correct material for the picking order. Additionally, the workstation transmits its location where the material has to be delivered. This information is necessary for the multi-criteria negotiation between the agents. The picking order also contains picking information, for example, the orientation of the material, if the workstation needs it. As response to the picking order, the selected PS-Agent sends a picking confirmation. This confirmation includes the available time slot and the location of the picking station.

\subsubsection{Inventory request}

WS-Agents create the inventory request and send it to the LA-Agents. The request contains the material ID, the necessary quantity, and the location and ID of the workstation or picking station, the material is intended for. Because it contains the material ID, only loading aids that contain this material will respond. The response the WSAgent gets contains the information whether the material in the right amount is available or not.

\subsubsection{Warehouse order}

The LA-Agents create a warehouse order and send it to the WH-Agent of the warehouse where it is located. If it is a retrieval order, it contains the time slot for the retrieval, as well as the current storage location. The WH-Agent sends a retrieval confirmation to the LA-Agent. This message includes the retrieval position as well as the time it is available for pick up by an AGV. The LA-Agent uses this information to create a transport order and sends it to the AGV-Agents.

If it is a storing process, the LA-Agent creates a warehouse order and the WH-Agent sets a storage location. Afterwards, the LA-Agent receives a storage confirmation with the date and the storage location. The LA-Agents saves the storage location for the retrieval process.

\subsubsection{Retrofitting order}

The AGV-Agents create the retrofitting order, if the AGV has to adapt his setup to certain criteria for a transport order. Therefore, the agent sends the order to the RS-Agent. The message includes the setup ID for the setup that is necessary and the time slot for the retrofitting. Additionally, it contains the location of the AGV, which is used in the multi-criteria negotiation of the RS-Agents. As a response, the AGVAgent receives a retrofitting confirmation from the selected RS-Agent. This includes the location of the setup station and the time the AGV has to be there.

\subsubsection{Transport order}

LA-Agents create and send transport orders to the AGVAgents. The orders include the specific loading aid ID, which is used by the loading aids to identify themselves. 
Besides that, the LA-Agent includes its location, the time slot for the execution, transport criteria, and the destination. Transport criteria are, for example, weight or dimensions of the loading aid. The possibility to specify and extend the information exists, for example, if the load is fragile.

\subsubsection{Sequence number}

The sequence number differentiates between current messages and outdated messages. Therefore, it shows the validity of the containing messages. The number eliminates through its structure the count to infinity problem. It works as follows: The sender of a message adds a sequence number. With every additional transmission of the identical information from the same sender, the number is increased by two. A detailed explanation of this procedure will be in Section 3.1 .8 of the error message. Higher sequence numbers imply information that is more up to date, which spread throughout the CPPS and have priority over older information. Due to this, the count to infinity problem does not exist anymore. The receiver of a message refreshes their information and only reacts, if the message has a higher sequence number. Otherwise, the receiver ignores the message. To create a reference to an order, every message includes a specific order ID .

\subsubsection{Error message}

Every entity of the CPPS can send and receive an error message. Either all entities receive the message or the receiver is specific. For example, if the production supply is not on time, not all entities receive the message. Only the production planning receives the message for a new allocation of the production order, whereas a failure of an entity, for example, a WS-Agent needs to be sent to every entity, because the AGV-Agent, the RS-Agent, the LAAgent, and the WH-Agent could be affected by the failure. Therefore, the cause of the error is decisive for the receiver of the error message. Thus, the message contains the error message and therefore the reason for the failure.

An error message from an entity can be sent out of two different situations. The first one is that the affected entity is still able to send an error message. In this case, the entity creates the error message itself and sends a message that all affected entities notice. The error message gets also a sequence number which is increased by two such as in a regular case. The second case is that an entity is not able to distribute the error message itself within the network. Then, another entity that notices the error can distribute the message.

An entity will send an error message, as soon as it recognizes the failure of another entity and no error message is in the system. For example, information is missing after a predefined time period. In this case, the entity only increases the sequence number by one. This is the only case where an entity does not increase its sequence number itself. Because the new sequence number is higher than the previous sent message, the information spreads within the whole CPPS. This makes sure that every other entity is informed about the error.

Furthermore, if the disrupted entity is repaired, it can immediately get back into the communication. The entity just increases the previous sent sequence number by two, and then, the new sequence number is higher than the sequence number from the error message sent by another entity.

\subsection{Qualitative evaluation of realProS}

The qualitative evaluation of realProS is based on three different qualitative objectives. These objectives are scalability, flexibility, and universality. In the following paragraphs, these three objectives are elaborated.

Scalability describes the ability of a concept to adapt to workload fluctuations by adding or removing entities [13]. The exchange of information between the entities should ensure that the information spreads through liquidated entities. This should ensure that the existing information and knowledge of agents are also made available to newly added entities. The concept considers the failure of entities. It defines possible situations and shows potential solutions. Additional entities can be added. Because the concept uses a multi-agent system, the information exchange and the integration of new entities of already existing entity classes are possible. For example, an entity can be added to the CPPS again after a defect through the initialization state. This is also possible for new entities, if they can be assigned to an already existing entity class of the concept.

Flexibility reflects the ability to react to changes in the environment, within certain predefined limits [14]. Concerning the definition, realProS is flexible. However, the concept only defines common failures, presents solutions to these failures, and considers possible process changes of the entities. Only the defined solution from the work for the failure is taken into account and the problem solved accordingly.

Universality aims to be able to apply a concept in general and adapt it to the given circumstances, not focusing on a specific area [15]. With the structure of the concept, universality is achieved. There is no specific area or industry sector considered. The concept can be extended through additional entity classes for the specific application.

\section{Implementation of realProS and a reference system}

In order to compare the realProS with a classical production supply by kanban, a comparison of both took place. This 
Fig. 2 Research Lab TU

Dortmund

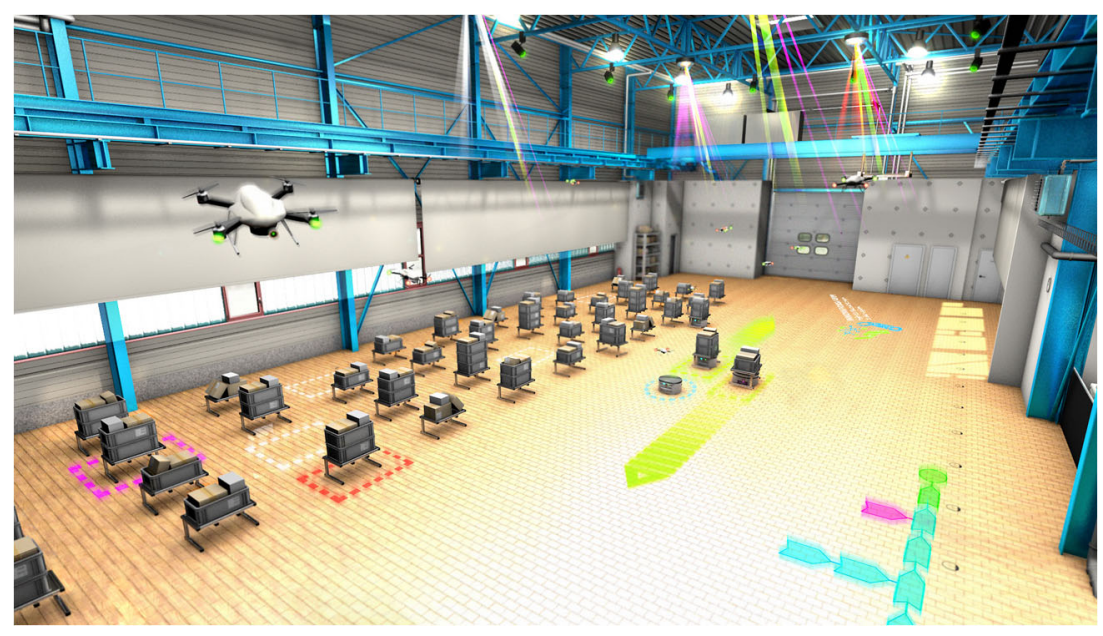

happened in the research lab of the Chair of Material Handling and Warehousing at the TU Dortmund University. The research lab is equipped with several industry 4.0 technologies, for example, automated guided vehicles (AGVs), motion capturing cameras, and intelligent small loading carriers, shown in Fig. 2. For the validation in the research lab, an area of $300 \mathrm{~m}^{2}$ is available to build an example production. Within this example production, the developed production supply processes are shown for the assembly of a sample product.

The product characteristics require simple customization. For this reason, a Lego drone is selected as an example. Different colors and two different models achieve the customization. In total, there are 24 different setups. The product consists of 27 or 38 parts depending on the setup. The assembly is divided into nine steps, which are fulfilled by nine workstations. The assembly process differentiates between the two production supply concepts.

The objective of this implementation is to proof the feasibility of the realProS concept, as well as the advantages and disadvantages compared with a classical production supply. For a quantitative comparison of the two concepts, four characteristics were measured: the energy consumption of the forklift trucks and AGVs, the amount of logistics employees, the size of the logistics area, and the required time. Table 1 shows the measured characteristics for both concepts.

The following sections present the implementation of the classical production supply and the implementation of the realProS. Then, an efficiency evaluation of both concepts based on the measured characteristics takes place.

\subsection{Implementation of classical production supply concept}

The classical production supply concept is realized by a storage area, an assembly line, a milk run train, and two employees. One employee is required to operate the milk run train and one for the storage and retrieval of the material in the storage area. The assembly line consists of nine workstations. Each workstation represents one step. The assembly line knows the production plan before the assembly starts. Hence, the production supply knows the quantity and the time when the parts have to be at a working station. Each workstation has a supermarket for the immediate production supply. The dimensions of these supermarkets are according to the demand of the workstation. Kanban controls the resupply of the supermarkets and a milk run train delivers the parts from the storage area. The electric tractor LTX 20 from Still operates the milk run. The dimensioning of the milk run is in accordance with Günthner et al. [16]. The following parameters are the result of the dimensioning. There are three stop points and nine staging areas. The maximum capacity of a milk run is 35 small load carriers with a utilization rate of $88 \%$. The milk run achieves a transport volume of 174 small loading carriers with six tours per hour.

With the previous described components for the production supply, the following process was developed. When the inventory of a material reaches the reorder point in a supermarket, the kanban system triggers the resupply. The employee in the storage area then gets a retrieval order for

Table 1 Characteristics of implemented concepts

\begin{tabular}{lll}
\hline Parameters & Classical concept & realProS \\
\hline Forklift trucks & 1 & 0 \\
AGVs & 0 & 10 \\
Energy consumption & $0.2 \mathrm{kWh}$ & $0.02 \mathrm{kWh}$ \\
Employees & 2 & 0 \\
Area & $50.56 \mathrm{~m}^{2}$ & $37.1 \mathrm{~m}^{2}$ \\
Time & $0.17 \mathrm{~h}$ & $0.27 \mathrm{~h}$ \\
\hline
\end{tabular}


the needed material. The retrieval of the small load carrier from the storage area is processed by the man to goods principle. Afterwards, the employee puts the small load carrier on the transfer area for the next milk run tour. The milk run delivers the small load carrier to the supermarket. The resupply of the storage area is not considered in the validation because it does not differentiate between the two alternative concepts.

\subsection{Implementation of real-time production supply concept}

The second concept that was validated is the realProS concept that was described in Section 3. This system consists of a different setup than the previous, because the concept was developed for the environment of cyberphysical production systems. The following components are used for the setup: a storage area, ten AGVs, and nine workstations. In this concept, several workstations can do several work processes. So, the production plan is not known before the assembly starts. The unfinished good requests only the next step and then selects the best workstation. The result of this flexible production sequence is that the location where the raw materials for each assembly step are needed is known just shortly before the assembly starts. Because of this problem, the realProS was developed. The storage area supplies the workstation now directly with AGVs and without any intermediate inventory.

Since the realProS is a universal applicable concept, it has to be customized for the individual application. The result is the following process for the new concept.

At first, the unfinished good requests the next assembly step and selects the best workstation. This workstation then requests the required raw materials. Then, the warehouse management system checks whether the stock is in the storage area or in circulation. After the material is located, a transport request is sent to the AGVs and the AGVs select the best one. Then, the selected AGV transports the material to the workstation and the workstation employee takes out the needed amount. Afterwards, the AGV transports the material back to the storage area or to another workstation, if it already requested the material.

\subsection{Efficiency evaluation}

The following analysis compares the efficiency of the two logistic concepts described above. For this purpose, a key performance indicator system is first presented, which represents the efficiency of production processes. This system is called process status indicators (PSIs). The PSIs are used because they are able to describe the efficiency of a production process depending on any number of input and output variables. Furthermore, they are scalable so that they can be applied to any hierarchical level of a production system. A single operating resource and the process executed on it can be mapped. For example, this can be the transport of a product within the factory using a forklift truck. But also, the efficiency of a factory can also be represented. In this way, the entire production system is seen as one process, so that the efficiency of the complete production is represented $[17,18]$.

\subsubsection{Indicator system}

The process status indicators consist of three indicators, which are summarized in Fig. 3. Only time-continuous process variables are used to calculate the key performance indicators. Typical variables are energy requirements, as electric or thermal energy, or the number of processed products. Furthermore, variables such as the working time of employees or machines can also be considered $[17,18]$.

The first indicator is the Energy Performance Indicator (EPI) which is calculated as

$E P I_{P P_{k}, I n_{j}, O u t_{j}}=\frac{O u t_{j}}{I n_{i}}$

The EPI describes the relation between one input variable $I n_{i}$ and one output variable $O u t_{j}$ regarding the considered production process $P P_{k}$. Consequently, for a process with several inputs and outputs, there is a corresponding number of EPIs to represent all relations between the process parameters [17, 18]. For example, an EPI results from the ratio of transported products $Q_{\text {out }}$ to the electric energy

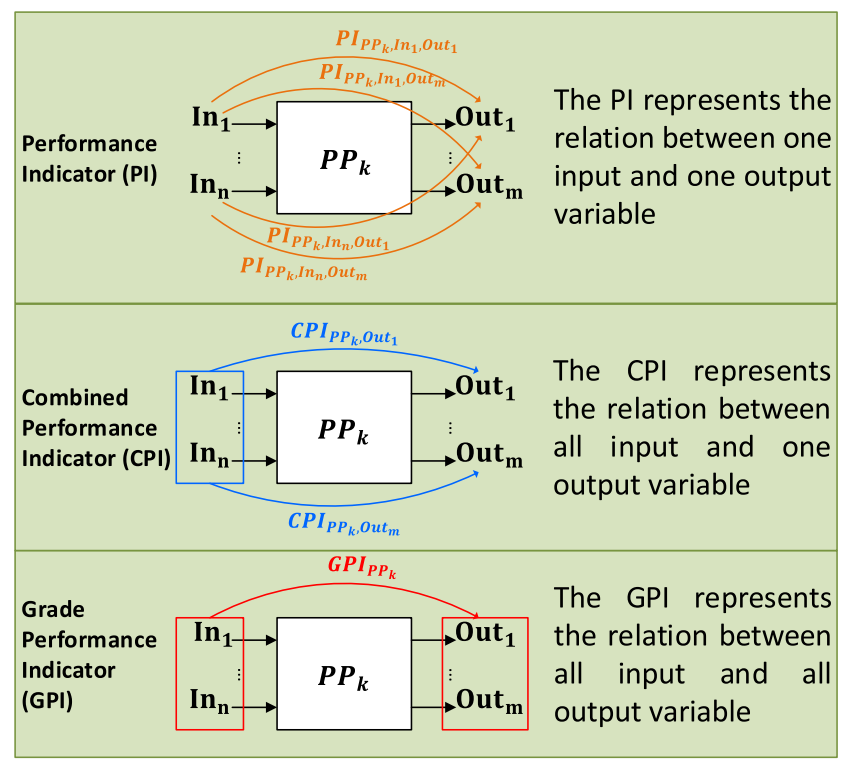

Fig. 3 Overview of the three process status indicators 
consumption $E_{\mathrm{el}}$ of a logistic process carried out by an automated guided vehicle (AGV) as

$E P I_{\mathrm{AGV}, E_{\mathrm{el}}, Q_{\mathrm{out}}}=\frac{Q_{\mathrm{out}}}{E_{\mathrm{el}}}$

Consequently, this indicator receives the individual unit pieces per $\mathrm{kWh}$. Each EPI has a unique unit depending on the inputs and outputs considered [17, 18].

The second step involves the calculation of the Combined Energy Performance Indicator (CEPI), which represents the relation between all input variables and one output variable of the considered production process $P P_{k}$. The CEPI is calculated as

$$
C E P I_{P P_{k}, \text { Out }_{j}}=\frac{1}{\sum_{i=1}^{n} \frac{b_{i}}{E P I_{P P_{k}, \text { In }_{j}, \text { Out }_{j}}}}
$$

The respective EPIs are weighted with a rating factor $b_{i}$, to assess the influence of the separated inputs on the efficiency. An economic assessment is chosen, as this is the most relevant approach for companies. This procedure is necessary because not every input variable has the same effect on the efficiency [17, 18]. A typical example is the relationship between the needed electrical energy and the working time of employees required for a process. Usually the electrical energy has a much smaller influence, as it is economically cheaper than the working time of employees. In this investigation, the rating factor for electric energy is weighted with $b_{\mathrm{el}}=0.16 € / \mathrm{kWh}$ [17-19]. In comparison, the rating factor of the employee working time is chosen to $b_{\mathrm{Emp}}=19.50 € / \mathrm{h}[20,21]$. In addition, the working time of machines can be assessed by the machine hour rate. For example, a rating factor $b_{\mathrm{AGV}}=1.75 € / \mathrm{h}$ is assumed for the use of automated guided vehicles.

Finally, the Grade Energy Performance Indicator (GEPI) is determined, which describes the relation between all input and all output variables of a production process $P P_{k}$. The calculation of the GEPI results from the relationship between the current state of the production process and a reference state of this process. The equation for the GEPI is

$G E P I_{P P_{k}}=\frac{\sum_{j=1}^{m} \frac{\text { Out }_{j}}{\operatorname{CEPI}_{P P_{k}, \text { out }_{j}, \text { ref }}}}{\sum_{j=1}^{m} \frac{\text { Out }_{j}}{C E P I_{P P_{k}, \text { Out }_{j}}}}$

By default, the considered process is analyzed under ideal conditions to determine the reference values $C E P I_{P P_{k}, \text { Out }_{j} \text {, ref }}[17,18]$. For the comparison of two different production systems, it is advisable to use one of the two systems as the reference system. In this way, the two systems are set directly in relation to each other. In this case, the GEPI of the reference system becomes one in principle. If the GEPI of the other system is greater than one, the selected reference system is less efficient than the other system.

In the following investigation, the classic intralogistics principle carried out by an employee and forklift truck is used as reference system. Accordingly, the reference value $C E P I_{\text {Classic, } \text { Out }_{j} \text {, ref }}$ is determined by considering the classical system and is set in relation to the new production supply concept realProS. Correspondingly, the GEPI of the new concept is calculated as

$G E P I_{\text {realProS }}=\frac{\sum_{j=1}^{m} \frac{\text { Out }_{j}}{C E P I_{\text {Classic }, \text { Out }_{j}, \text { ref }}}}{\sum_{j=1}^{m} \frac{\text { Out }_{j}}{C E P I_{\text {realProS }, \text { out }_{j}}}}$

\subsubsection{Comparison of the two production supply concepts}

The input and output variables for the two production systems which differ in their production supply concept are listed in Table 2. The efficiency investigation considers as input variables the electrical energy $E_{\mathrm{el}}$, the number of employees $N_{\text {Emp }}$ and number of forklift trucks $N_{\text {FT }}$ or the number of AGVs $N_{\mathrm{AGV}}$, the needed area of the production system $A_{\mathrm{PS}}$, and the required processing time $T_{\text {Pro }}$. For the rating of the processing time, it should be noted that it differs in the two systems. The output quantity $Q_{\text {out }}$ of drones produced is set equal for both systems. Each system assembles 20 drones, which results in different processing times. In the classic system, the processing time is assessed by the use of two employees, each of them receives an hourly wage of $19.50 € / \mathrm{h}$ and the machine hour rate of the used forklift truck which is set to $22.45 € / \mathrm{h}$. Consequently, the processing time of the classic system is rated with $61.45 € / \mathrm{h}$. By using the new system, the processing time is assessed by a rating factor of $17.50 € / \mathrm{h}$ depending on the number of AGVs.

Table 2 Characteristics of the two concepts

\begin{tabular}{lll}
\hline & Classical concept & realProS \\
\hline$E_{\text {el }}$ & $0.2 \mathrm{kWh}$ & $0.02 \mathrm{kWh}$ \\
$N_{\text {Emp }}$ & 2 & 0 \\
$N_{\mathrm{FT}}$ & 1 & 0 \\
$N_{\mathrm{DTV}}$ & 0 & 10 \\
$A_{\mathrm{PS}}$ & $50.565 \mathrm{~m}^{2}$ & $37.13 \mathrm{~m}^{2}$ \\
$T_{\text {Pro }}$ & $0.17 \mathrm{~h}$ & $0.27 \mathrm{~h}$ \\
$Q_{\text {Out }}$ & 20 & 20 \\
$b_{\text {el }}$ & $0.16 € / \mathrm{kWh}$ & \\
$b_{\text {Emp }}$ & $19.50 € / \mathrm{h}$ & \\
$b_{\mathrm{FT}}$ & $22.45 € / \mathrm{h}$ & \\
$b_{\mathrm{A}}$ & $4 € / \mathrm{m}^{2}$ & \\
$b_{\text {AGV }}$ & $1.75 € / \mathrm{h}$ & \\
\hline
\end{tabular}


Table 3 Resulting process status indicators for the two concepts

\begin{tabular}{lll}
\hline Indicator & Classical concept & realProS \\
\hline$E P I_{E_{\mathrm{el}}, Q_{\text {out }}}$ & 100 & 1000 \\
$E P I_{A_{\mathrm{PS}}}, Q_{\text {out }}$ & 0.3955 & 0.5388 \\
$E P I_{T_{\mathrm{Pro}}, Q_{\text {out }}}$ & 117.6471 & 74.0741 \\
$C E P I_{Q_{\text {out }}}$ & 0.0940 & 0.1305 \\
$G E P I$ & 1 & 1.3886 \\
\hline
\end{tabular}

The characteristics in Table 2 do not directly indicate which of the two systems is more efficient. The realProS concept requires significantly less electrical energy and less space, but it takes clearly longer to finish the production order. For a more precise evaluation of the efficiency, the process status indicators are listed in Table 3. Since the old system is used as a reference system, its $G E P I_{\text {Classic }}$ is 1 . In comparison, the GEPI of the innovative system becomes $G E P I_{\text {realProS }}=1.39$, indicating that the system using realProS is more efficient.

The increase in efficiency results on the one hand from the reduction of the two input variables area $A_{\mathrm{PS}}$ and electrical energy $E_{\mathrm{el}}$. On the other hand, the monetary assessment per hour regarding the processing time for the realProS is significantly lower compared with the classical concept that uses the kanban and milk run principle. However, for the new concept, it has to be considered that the processing time $T_{\text {Pro }}$ rises, which leads to a longer delivery time for the customers.

\section{Conclusion}

This paper proposes a novel real-time production supply concept for cyber-physical production systems (CPPS) called real-time production supply concept (realProS). The objective of the concept is to be able to handle the new challenges the production supply faces from the changing requirements of the customer. The necessity of such a concept arises from the fact that current production supply concepts are not designed to meet the dynamic requirements of industrial development. Current research projects only concentrate on the aspects of hardware development or the further development of classic concepts such as kanban or milk run. However, the different aspects are only considered separately from each other. The realProS concept, in contrast, is a comprehensive concept that takes all aspects into account.

RealProS decision-making has an nondeterministic approach. Because of this, every time a material is needed in the production, realProS determines which entities are the most suited one at the given time. It decides if a picking order is necessary, which stock of the needed materials should be used and which AGV is the best to transport the materials to the workstation. This decision is based on various parameters which are based on the application scenario. In the presented scenario, the decision is based on time and costs.

The concept can be evaluated based on qualitative and quantitative aspects. Scalability, flexibility, and universality are qualitative aspects companies try to achieve, because of the changing environment. realProS can fulfill these requirements. In terms of scalability, the realProS concept is able to react to fluctuations in the workload. This can be done by adding or removing elements in each entity. If an element is added, it will be available and will join the communication for the next decision. If an element is removed, it will not be available anymore and the next decision takes place without it. The flexibility is achieved by the ability of reacting to predefined changes. This means realProS is capable to react to occurring errors and generates suitable solutions. For example, if an automated guided vehicle (AGV) is broken, the concept identifies the error by itself and all the open tasks of this AGV will be renegotiated that other AGVs take over the tasks from the broken AGV. The new approach has been developed independently of specific areas or industrial sectors, which enables it to be applied universally. This means the concept can handle different requirements from different manufacturing settings, and therefore, universality is achieved. For further investigations, scalability, flexibility, and universality should be evaluated quantitatively for a more precise understanding of the system.

For the quantitative aspect, the concept was implemented and compared with a reference system based on their efficiency. For this comparison, the implementation took place in the research lab of the Chair of Material Handling and Warehousing at the TU Dortmund University. The efficiency analysis of both concepts is carried out with an innovative key performance indicator system called process status indicators. This indicator system is able to describe production processes with any number of inputs and outputs, whereby an economic assessment of the input variables takes place in order to reflect their influence on the efficiency. The investigation shows that the new concept realProS operates more efficiently than the classic supply concept. Thereby, the classic concept based on the use of kanban and milk run. This efficiency improvement is based on a reduction of the electrical energy consumption and the required area. In addition, the new concept causes lower hourly costs, since no more employees are needed and the machine hour rate for AGVs is significantly lower. However, it must be taken into account that the processing time of the new concept is longer in relation to the classic variant. This must be considered especially in production planning and with regard to adherence to delivery dates. 
Due to the scope, this paper focuses on a proof of concept, containing a first quantitative comparison. The figures for this comparison were recorded to get a first overview if the concept can compete with classical systems. For future research, these figures need to be recorded on a more profound level to get a more detailed analysis. Furthermore, the concept in this paper presumes the materials are in stock. A procurement system for the requirements of the concept has to be identified and implemented into the CPPS environment.

By using the newly developed production supply concept realProS, intralogistics systems are able to react to changes in real time. As a result, logistics can be designed more flexibly and can respond to the increasingly dynamic requirements of industrial development. In conclusion, realProS is a production supply concept that not only has a higher efficiency. As stated before, it also enables flexibility, scalability, and universality.

Funding Information Open Access funding provided by Projekt DEAL.

Open Access This article is licensed under a Creative Commons Attribution 4.0 International License, which permits use, sharing, adaptation, distribution and reproduction in any medium or format, as long as you give appropriate credit to the original author(s) and the source, provide a link to the Creative Commons licence, and indicate if changes were made. The images or other third party material in this article are included in the article's Creative Commons licence, unless indicated otherwise in a credit line to the material. If material is not included in the article's Creative Commons licence and your intended use is not permitted by statutory regulation or exceeds the permitted use, you will need to obtain permission directly from the copyright holder. To view a copy of this licence, visit http://creativecommons. org/licenses/by/4.0/.

\section{References}

1. Koren Y (2010) The global manufacturing revolution: productprocess-business integration and reconfigurable systems. Wiley series in systems engineering and management. Wiley

2. Bauernhansl T, ten Hompel M, Vogel-Heuser B (eds) (2014) Industrie 4.0 in Produktion, Automatisierung und Logistik. Springer, Fachmedien

3. Klug F (2010) Logistikmanagement in der Automobilindustrie. Springer, Berlin

4. Conze MH (2014) Integrierte Abruf- und Transportsteuerung für lagerhaltige Teileumfänge zur schlanken Versorgung der Automobilmontage. Technical University of Munich, Ph.D. thesis

5. Bullinger HJ, Lung MM (1994) Planung der Materialbereitstellung in der Montage. Teubner, Stuttgart Germany

6. Ohno T (1988) Toyota production system: beyond large-scale production. Productivity Press, Cambridge
7. Greenwood AG, Kluska K, Pawlewski P (2017) A multi-level framework for simulating milkrun, in-plant logistics operations. In: Bajo J (ed) Highlights of practical applications of cyberphysical multi-agent systems, vol 722. Springer International Publishing, Cham, pp 209-220

8. Bortolini M, Ferrari E, Gamberi M, Pilati F, Faccio M (2017) Assembly system design in the Industry 4.0 era: a general framework. IFAC-PapersOnLine 50(1):5700-5705

9. Wang W, Zhang Y, Zhong RY (2020) A proactive material handling method for CPS enabled shop-floor. Robot Comput Integr Manuf 61:101849

10. Weichert F SMART FACE - Smart Micro Factory für Elektrofahrzeuge mit schlanker Produktionsplanung: Cyber Physical Systems und Industrie 4.0: Wie Produktion, Produkte und Dienste im multidimensionalen Internet der Zukunft funktioniert

11. Trenkle A, Seibold Z, Stoll T (2013) Safety requirements and safety functions for decentralized controlled autonomous systems. In: 2013 XXIV International conference on information, communication and automation technologies (ICAT). Sarajevo, Bosnia and Herzegovina, pp 1-6

12. Vojdani N, Knop M (2014) Adaptive Materialbereitstellung in flexiblen Produktionssystemen auf Grundlage einer agentenbasierten Transportsteuerung. Logistics Journal: Proceedings 2014(01)

13. Koren Y, Wang W, Gu X (2017) Value creation through design for scalability of reconfigurable manufacturing systems. Int J Prod Res 55(5):1227-1242

14. Sethi A, Sethi S (1990) Flexibility in manufacturing: a survey. International Journal of Flexible Manufacturing Systems 2(4)

15. Popp J (2018) Neuartige Logistikkonzepte für eine flexible Automobilproduktion. Ph.D. thesis University of Stuttgart

16. Günthner WA (2013) Schlanke Logistikprozesse: Handbuch für den Planer. Springer, Berlin

17. Meißner M, Massalski L, Wirtz A, Wiederkehr P, Myrzik J (2018) Representation of energy efficiency of energy converting production processes by process status indicators. In: 12th CIRP conference on intelligent computation in manufacturing engineering. Gulf of Napels, Italy, pp 221-226

18. Meißner M, Rehtanz C, Myrzik J (2019) Application of the non-linear optimization algorithm differential evolution for optimization of manufacturing systems regarding their enery efficiency. In: Proceedings of the 2019 Winter simulation conference. National Harbor, Maryland, pp 21542165

19. Federal Ministry for the Environments (2014) Nature Conservation, Building and Nuclear Saftey: Entwicklung einer Mehodik zur Aufstellung von Energiekennzahlen zur Steigerung der Energieeffizienz in Unternehmen

20. Statistisches Bundesamt (Destatis) (2017) Verdienste auf einen Blick Statistisches Bundesamt (Destatis)

21. Statistisches Bundesamt (Destatis) (2019) Earnings by economic activity. https://www.destatis.de/EN/Themes/Labour/Earnings/ Earnings-Earnings-Differences/Tables/quaterly-earnings.html

Publisher's note Springer Nature remains neutral with regard to jurisdictional claims in published maps and institutional affiliations. 